\title{
Outputs from "Spectrum-Imaging and Hyperspectral Data Analysis 2003", an MAS Special Topics Workshop
}

\author{
John Henry J. Scott *, Ken Livi **, Ed Vicenzi *** \\ * Surface and Microanalysis Science Division, National Institute of Standards and Technology \\ (NIST), 100 Bureau Drive Stop 8371, Gaithersburg, MD 20899-8371 \\ ** Department of Earth and Planetary Sciences, 129 Olin Hall, 34th and North Charles Streets, \\ Johns Hopkins University, Baltimore, Maryland 21218 \\ *** National Museum of Natural History, Smithsonian Institution, Washington, D.C. 20560- \\ 0119
}

This talk will present results from a four-day Microbeam Analysis Society (MAS) Special Topics Workshop on spectrum-imaging and multispectral \& hyperspectral data analysis held at NIST in Gaithersburg, Maryland from April 28th through May 1st, 2003 [1]. The scope of the workshop included many forms of multispectral and hyperspectral data relevant to microbeam analysis, including STEM-based EELS and XEDS, energy-filtered TEM imaging, XEDS in the SEM, spectrum-imaging in mass spectrometry, and optical spectroscopies such as IR and cathodoluminescence [2]. The workshop was intended in part to capture information about the current state of hyperspectral techniques in microbeam analysis, identify significant needs and opportunities for progress, and produce a roadmap to guide future work.

To learn about the current state of the art in hyperspectral microanalysis, more than a dozen invited speakers were chosen based on their current involvement and progress in the field. Because the meeting was laid out on a workshop format instead of a symposium format, the invited speakers were asked to provide a tutorial-like foundation for the discussions and panel sessions included in the technical program. Although some of these speakers are participating in the current symposium at Microscopy and Microanalysis 2003, where it is appropriate important results from those speakers unable to travel to San Antonio will be recounted and pointers provided to the full detail of the original work.

Another aspect of the effort to capture the current state of the art focused on the importance of support from the commercial sector and included an assessment of the resources offered by vendors. About a dozen vendors participated in the workshop, either by manning an exhibit booth, presenting a scientific talk, or as a corporate sponsor of the meeting. A wide array of data acquisition, post-processing, and analysis tools are available off-the-shelf and all researchers in the field should be aware of the options open to them. In return, open discussions of end-user needs and wants provide vendors with important market data that help guide product development and shape the next generation of hyperspectral tools.

In addition to traditional invited and contributed talks and posters, the workshop included open discussions on several topics of interest to the larger hyperspectral microanalysis community. These discussions, all concerned with the future direction of work in the field, were semistructured brainstorming sessions designed to produce a consensus opinion or a draft of a 
strawman standard. Conclusions and outputs from the most important of these sessions will be presented along with progress reports on associated summary documents:

\section{Nomenclature}

A quick glance at the literature indicates examples of inconsistent nomenclature in multispectral and hyperspectral analysis. This is expected both because of the varied backgrounds of microanalysts who use these techniques, and because many of the techniques have been borrowed from more mature problem domains such as remote sensing, signal processing, geoscience, and others. To promote effective communication, this discussion attempted to find agreeable definitions of terms often used in hyperspectral microanalysis. Examples include the difference between hyperspectral and multispectral analyses, spectrum-image vs. imagespectrum, and BSQ vs. BIL vs. BIP band interleaving conventions [2].

\section{Information Management and File Formats}

The proliferation of sophisticated tools for processing hyperspectral microanalysis datasets has unfortunately been accompanied by a proliferation of incompatible file formats for storing and transmitting both the experimental data and metadata. The goal of this discussion was an outline of what an open, standardized file format for hyperspectral microanalysis data should include, how it should be implemented, and what toolset should be built to support it. Issues include the tradeoffs between binary $v s$. ASCII $v s$. structured (XML) formats, data mining and centralized repositories, and compatibility with existing hyperspectral formats in use outside of microanalysis.

\section{Roadmap}

A rapidly growing number of industries, technology sectors, and scientific efforts recognize the need for organized technology planning, especially the development of a technology roadmap. While an extensive document is not required here, it is helpful to have: (1) a clear picture of the needs that are driving microanalysis research and development into multidimensional techniques, (2) some understanding of the technology gaps currently limiting microanalysis capabilities in this area, and (3) a coherent plan for making decisions about the feasibility and priority of future work.

\section{References}

[1] more information about the workshop and its outputs can be found at http://www.cstl.nist.gov/div837/Division/meetings/Hyperspectral/Hyperspec.htm or http://www.microbeamanalysis.org/masjh/mastc/2003-hyperspectral.php [2] STEM = Scanning Transmission Electron Microscopy; EELS = Electron Energy-Loss Spectroscopy; XEDS = Energy-Dispersive X-ray Spectrometry; SEM = Scanning Electron Microscopy; IR = Infrared; BSQ = Band Sequential; BIL = Band Interleave by Line; BIP = Band Interleave by Pixel. 